\title{
Duration of anuria predicts recovery of renal function after acute kidney injury requiring continuous renal replacement therapy
}

Hee-Yeon Jung ${ }^{\star}$, Jong-Hak Lee ${ }^{\star}$ Young-Jae Park, Sang-Un Kim, Kyung-Hee Lee, Ji-Young Choi, Sun-Hee Park, Chan-Duck Kim, Yong-Lim Kim, and Jang-Hee Cho

Department of Internal Medicine, Kyungpook National University School of Medicine, and Clinical Research Center for End Stage Renal Disease, Daegu, Korea

Received: September 25, 2014

Revised : June 30, 2015

Accepted: July 4, 2015

\section{Correspondence to}

Jang-Hee Cho, M.D.

Division of Nephrology,

Department of Internal Medicine,

Kyungpook National University

School of Medicine, 680 Gukchae-

bosang-ro, Jung-gu, Daegu 41944,

Korea

Tel: $+82-53-420-6314$

Fax: +82-53-423-7583

E-mail:jh-cho@knu.ac.kr

*These authors contributed equally to this work.
Background/Aims: Little is known regarding the incidence rate of and factors associated with developing chronic kidney disease after continuous renal replacement therapy (CRRT) in acute kidney injury (AKI) patients. We investigated renal outcomes and the factors associated with incomplete renal recovery in AKI patients who received CRRT.

Methods: Between January 2011 and November 2013, 408 patients received CRRT in our intensive care unit. Of them, patients who had normal renal function before AKI and were discharged without maintenance renal replacement therapy (RRT) were included in this study. We examined the incidence of incomplete renal recovery with an estimated glomerular filtration rate $<60 \mathrm{~mL} / \mathrm{min} / 1.73 \mathrm{~m}^{2}$ and factors that increased the risk of incomplete renal recovery after AKI.

Results: In total, 56 AKI patients were discharged without further RRT and were followed for a mean of 8 months. Incomplete recovery of renal function was observed in 20 of the patients (35.7\%). Multivariate analysis revealed old age and long duration of anuria as independent risk factors for incomplete renal recovery (odds ratio [OR], 1.231; 95\% confidence interval [CI], 1.041 to 1.457; $p=0.015$ and OR, 1.064; $95 \%$ CI, 1.001 to $1.131 ; p=0.047$, respectively). In a receiver operating characteristic curve analysis, a cut-off anuria duration of 24 hours could predict incomplete renal recovery after AKI with a sensitivity of $85.0 \%$ and a specificity of $66.7 \%$.

Conclusions: The renal outcome of severe AKI requiring CRRT was poor even in patients without further RRT. Long-term monitoring of renal function is needed, especially in severe AKI patients who are old and have a long duration of anuria.

Keywords: Acute kidney injury; Anuria; Chronic kidney disease; Continuous renal replacement therapy; Renal recovery

\section{INTRODUCTION}

Acute kidney injury (AKI) is a common clinical disorder, defined by an abrupt increase in serum creatinine or a decreased urine output. Clinical manifestations of AKI vary from an asymptomatic decline in renal function to anuric kidney injury, requiring renal replacement therapy (RRT) [1,2]. Among patients with AKI undergoing RRT, those who are hemodynamically unstable require continuous renal replacement therapy (CRRT) rather than intermittent hemodialysis, regardless of the presence of pre-existing comorbid conditions. The number 
of critically ill patients with AKI requiring CRRT is increasing, and the mortality of patients with severe AKI is as high as $80 \%$, despite advances in renal replacement techniques and critical care medicine [3].

Aside from mortality, renal outcomes after AKI are also important. Previous reports demonstrated that survivors after AKI have an increased risk of end-stage renal disease (ESRD), although any causal relationship and the pathogenesis remain unclear [4,5]. A recent study showed that renal recovery status after AKI was also associated with progression to chronic kidney disease (CKD) and ESRD, as well as patient survival [6]. Moreover, while AKI leads to CKD and ESRD, large retrospective cohort studies have also reported that CKD is a risk factor for the development of AKI [4,7].

Although concerns regarding the bidirectional relationship between AKI and CKD are increasing, the incidence rate of and the factors predicting persistent renal injury in critically ill patients with AKI receiving CRRT are not well known. Identifying risk factors leading to incomplete renal recovery after AKI is crucial for predicting outcomes in survivors of AKI and reducing the burden of CKD in the general population. In this study, we investigated renal outcomes and the factors associated with incomplete renal recovery in AKI patients who received CRRT.

\section{METHODS}

\section{Patients and data collection}

We investigated retrospectively all patients who received CRRT in our intensive care unit between January 2011 and November 2013. Patients with a pre-existing diagnosis of CKD (estimated glomerular filtration rate $[\mathrm{eGFR}]<$ $60 \mathrm{~mL} / \mathrm{min} / 1.73 \mathrm{~m}^{2}$ ) or ESRD before CRRT were excluded from the study. Other exclusion criteria were death and transfer to another medical center during CRRT. Only survivors with normal renal function prior to AKI who were discharged without maintenance RRT were included in this study. Normal renal function was defined as an eGFR $>60 \mathrm{~mL} / \mathrm{min} / 1.73 \mathrm{~m}^{2}$ in this study.

Patient data, including age, gender, baseline comorbidities, cause of AKI, duration of CRRT, duration of anuria, body mass index (BMI), baseline serum hemoglobin $(\mathrm{Hb})$, serum C-reactive protein $(\mathrm{CRP})$, serum creatinine
(Cr), eGFR, average mean arterial pressure (MAP), and volume balance, were retrieved from electronic medical records. Causes of AKI included ischemic acute tubular necrosis (ATN), infection-related ATN, and other ATN. Ischemic ATN was defined as AKI induced by hypovolemia, heart failure, or major surgery, all of which are associated with hypoperfusion. Cases occurring from both sepsis and AKI were defined as infection-related ATN. All other cases that triggered ATN were classified as 'other' ATN. Volume balance was calculated as the sum of input and output during the CRRT period. Serum $\mathrm{Cr}$ and eGFR were measured during the follow-up period after discharge.

The duration of anuria was defined as the period in which urine output was less than $0.5 \mathrm{~mL} / \mathrm{kg} / \mathrm{hr}$. Incomplete renal recovery was defined as eGFR $<60 \mathrm{~mL} /$ $\min / 1.73 \mathrm{~m}^{2}$ in the 3 months after discharge. In this study, eGFR was calculated using the simplified 'Modification of Diet in Renal Disease Study' equation.

\section{Continuous renal replacement therapy protocol}

Vascular access for CRRT was gained via the femoral or internal jugular vein. In all patients, continuous venovenous hemodiafiltration was performed using the PRISMA platform (Gambro, Hechingen, Germany). CRRT was initiated with a blood flow rate of 100 $\mathrm{mL} / \mathrm{min}$, which was increased gradually to $150 \mathrm{~mL} / \mathrm{min}$. CRRT was prescribed at a dose of more than $35 \mathrm{~mL} / \mathrm{kg} /$ hr, and Hemosol (Gambro) was replaced by the predilution method.

\section{Statistical analysis}

All enrolled patients were divided into complete and incomplete recovery groups, according to their eGFR values in the 3 months after discharge. Data are expressed as medians with ranges. Differences between groups were tested using independent-sample $t$ tests and chisquare tests, as appropriate. Factors that increased the risk of incomplete renal recovery after AKI were investigated by univariate logistic regression. Afterwards, multivariate logistic regression was performed in two models. In the adjusted model, all variables with a univariate $p<0.1$ were used in the logistic regression analysis to assess their independent influences on the clinical endpoint of "incomplete renal recovery." The fully adjusted model included all explanatory variables, such as age, 
gender, hypertension, diabetes mellitus, cause of AKI, duration of CRRT, anuria duration, initial serum $\mathrm{Hb}$, initial serum CRP, initial eGFR, average MAP, and volume balance, in the multiple logistic regression model. The ability of anuria duration to predict incomplete renal recovery was further analyzed with receiver operating characteristic (ROC) curves. Statistical analyses were performed using the SPSS version 18.0 (SPSS Inc., Chicago, IL, USA). The $p$ values of $<0.05$ were considered to indicate statistical significance.

\section{RESULTS}

In total, 408 patients were treated with CRRT during the study period. We excluded 300 patients who died during hospitalization or were lost to follow-up. Among the 108 surviving patients (26.5\%), 50 were diagnosed with CKD or ESRD. Of the $58 \mathrm{AKI}$ patients without previous CKD or ESRD, 36 (62.1\%) showed complete recovery to baseline renal function or an eGFR $>60 \mathrm{~mL} / \mathrm{min} / 1.73 \mathrm{~m}^{2}$. Moreover, 20 AKI patients (34.5\%) did not recover their baseline renal function and two patients (3.4\%) received maintenance dialysis after the withdrawal of CRRT, suggesting that $37.9 \%$ of the patients with AKI sustained a persistent renal injury. The data of 56 AKI patients who were discharged without further RRT were ultimately analyzed; these were divided into two groups based on their eGFR values 3 months after discharge (Fig. 1).

During a mean follow-up of 8 months, 20 of the 56 AKI patients (35.7\%) who received no further RRT recovered their renal function incompletely. The complete recovery group was younger than the incomplete recovery group $(56.3 \pm 15.2$ years vs. $66.4 \pm 11.4$ years, $p=0.014$ ). In addition, the complete recovery group showed shorter durations of anuria $(56.28 \pm 92.6$ hours vs. $201.3 \pm 219.8$ hours, $p=0.001)$ and CRRT ( $87.8 \pm 57.0$ hours vs. 140.2 \pm 78.9 hours, $p=0.006$ ) compared with the incomplete recovery group. Baseline conditions, causes of AKI, BMI, initial $\mathrm{Hb}$, initial CRP, initial $\mathrm{Cr}$, average MAP, and volume balance did not differ significantly between the two groups. Baseline characteristics are presented in Table 1.

Univariate logistic regression analysis showed that age (OR, 1.061; CI, 1.009 to 1.116; $p=0.021$ ), ischemic ATN as the cause of AKI (OR, 4.714; CI, 0.868 to 25.612; $p=$ 0.073), CRRT duration (OR, 1.012; CI, 1.003 to 1.022; $p=$

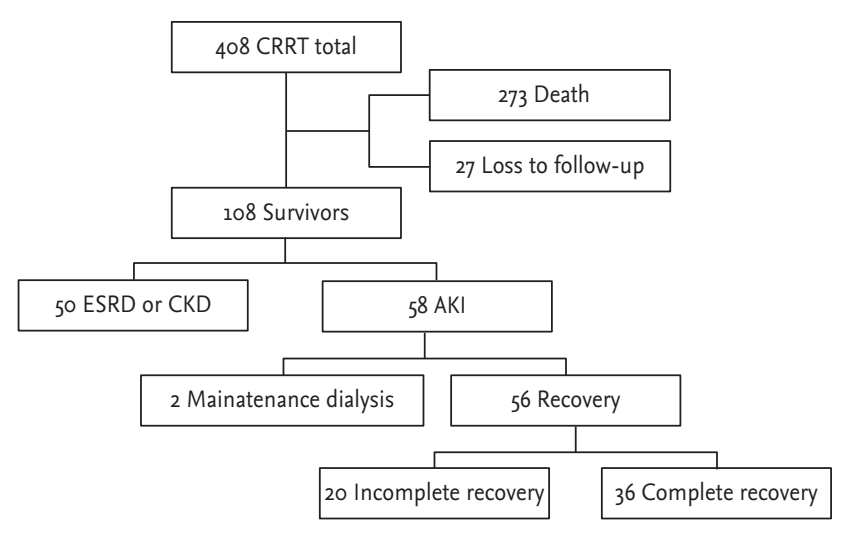

Figure 1. Study selection diagram. In total, 408 patients treated with continuous renal replacement therapy (CRRT) were initially enrolled. In the final analysis, 56 patients were divided into two groups (complete vs. incomplete renal recovery) based on their final estimated glomerular filtration rate values. ESRD, end-stage renal disease; CKD, chronic kidney disease; AKI, acute kidney injury.

0.013), and anuria duration (OR, 1.038; CI, 1.010 to 1.066; $p=0.007)$ were significant risk factors for incomplete recovery after AKI. Age (OR, 1.198; CI, 1.063 to 1.351; $p=$ 0.003 ), ischemic ATN (OR, 10.185; CI, 0.905 to 114.630; $p$ $=0.060$ ), CRRT duration (OR, 1.023; CI, 1.004 to 1.043; $p=0.020$ ), and anuria duration (OR, 1.059; CI, 1.007 to 1.114; $p=0.026$ ) remained significant after adjusting for age, ischemic ATN, infection-related ATN, CRRT duration, and anuria duration. Multivariate analysis results, fully adjusted for gender, diabetes, hypertension, cause of AKI, duration of CRRT, anuria duration, initial $\mathrm{Hb}$, initial CRP, initial eGFR, average MAP, and volume balance, revealed that only two factors, age (OR, 1.231; CI, 1.041 to $1.457 ; p=0.015)$ and duration of anuria $(\mathrm{OR}, 1.064$; CI, 1.001 to $1.131 ; p=0.047$ ), were significant risk factors for incomplete renal recovery after AKI (Table 2).

To compare the distribution of anuria duration between the two groups, we divided the duration of anuria into subcategories: shorter than 12 hours, 12 to 24 hours, 1 to 3 days, 3 to 10 days, and longer than 10 days. Compared with the incomplete recovery group, the complete recovery group included a significantly higher percentage of patients whose anuria duration was shorter than 12 hours (53\% vs. 10\%, $p=0.001$ ) (Fig. 2). Conversely, the percentage of patients with an anuria duration longer than 10 days was significantly higher in the incomplete recovery group than in the complete recovery group (30\% vs. $6 \%, p=0.023$ ) (Fig. 2 ). 


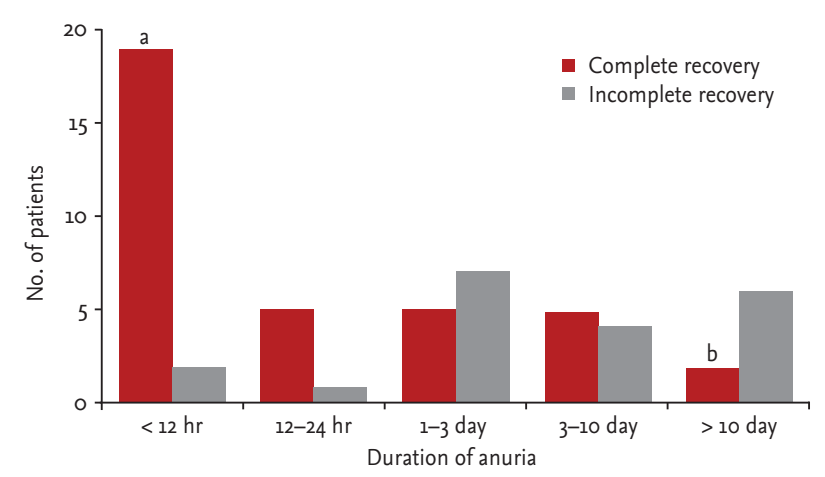

Figure 2. Comparison of anuria duration between the complete and incomplete recovery groups. The complete recovery group showed a significantly higher percentage of patients whose anuria duration was shorter than 12 hours compared with the incomplete recovery group. Conversely, the incomplete recovery group showed a significantly higher percentage of patients whose anuria duration was longer than 10 days compared with the complete recovery group. ${ }^{\mathrm{a}} \mathrm{p}$ $=0.001,{ }^{b} p=0.023$, compared with the incomplete recovery group.

ROC curve analysis showed that the area under the curve of anuria duration for predicting incomplete recovery of renal function after AKI was 0.776 , indicating that duration of anuria could be considered a fair predictor of incomplete renal recovery [8]. A cut-off anuria duration of 24 hours could predict incomplete renal recovery with a sensitivity of $85.0 \%$ and a specificity of $66.7 \%$ (Fig. 3).

\section{DISCUSSION}

In this study, we investigated factors associated with renal outcome in AKI patients treated with CRRT. Among the surviving severe AKI patients, approximately 37.9\% showed persistent renal injury, requiring maintenance dialysis or incomplete recovery with a GFR $<60 \mathrm{~mL} /$ $\min / 1.73 \mathrm{~m}^{2}$. We found that a long duration of anuria was an independent risk factor for incomplete recovery of renal function after CRRT. Additionally, our data suggest that anuria lasting for more than 24 hours is a good predictor of incomplete renal recovery. To our knowledge, this is the first study to report an association of anuria duration with renal recovery in AKI patients treated with CRRT.

As the incidence of AKI has increased over the past 20

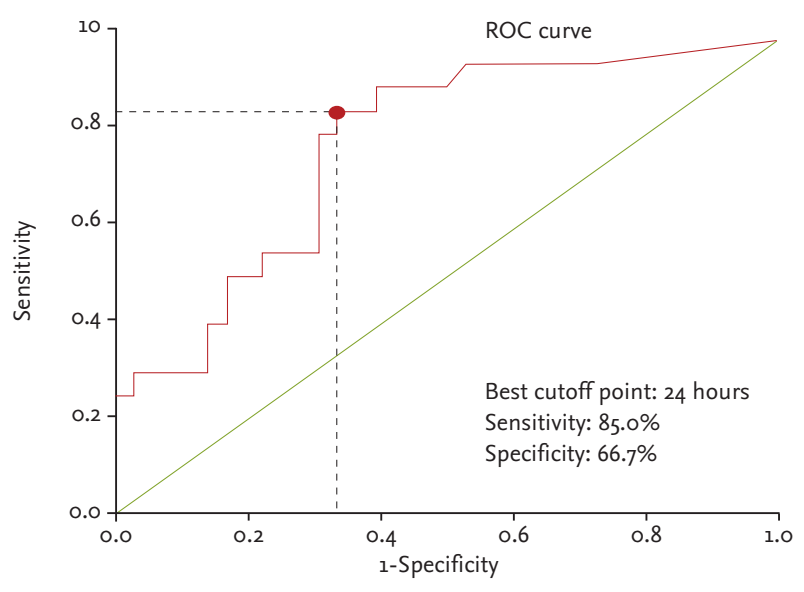

Figure 3. Receiver operating characteristic (ROC) curve for duration of anuria. The area under the curve of anuria duration for predicting incomplete renal recovery after acute kidney injury was 0.776 . A cut-off anuria duration of 24 hours could predict incomplete renal recovery with a sensitivity of $85.0 \%$ and a specificity of $66.7 \%$.

years, the incidence of AKI requiring CRRT, the most severe form of AKI, has also been reported to be increasing rapidly, averaging a 10\% increase per year $[9,10]$. Recent epidemiological studies have shown that AKI itself could increase the risk of developing incident CKD and further ESRD [4,5,11]. Even full recovery to normal renal function after AKI was associated with an increased risk of CKD [12]. It is evident that patients with AKI share several common risk factors: obesity, metabolic syndrome, diabetes, hypertension, cardiovascular disease, and microalbuminuria, factors that also increase the risk of CKD development [13].

However, the factors thought to be associated with incomplete renal recovery in AKI patients remain controversial. Schmitt et al. [14] performed a meta-analysis of 17 studies involving 5,529 patients to examine the recovery of kidney function after AKI. The studies showed significant heterogeneity in the definition of renal recovery, comorbid factors, study design, and length of follow-up. As a result, with the exception of age older than 65 years, no significant risk factor was identified. The findings from studies of AKI patients who received RRT are also very heterogeneous, making it difficult to draw conclusions regarding any predictor of renal recovery. Schiffl [15] reported that neither clinical features nor dialysis duration were associated with partial recovery in a single-center prospective study. Similarly, Allegretti 
Table 1. Baseline characteristics of AKI patients treated with CRRT

\begin{tabular}{|c|c|c|c|}
\hline Characteristic & Complete renal recovery $(\mathrm{n}=36)$ & Incompleterenal recovery $(\mathrm{n}=20)$ & $p$ value \\
\hline Age, yr & $56.3 \pm 15.2$ & $66.4 \pm 11.4$ & 0.014 \\
\hline Male sex & $23(63.9)$ & $9(45 \cdot 0)$ & 0.171 \\
\hline \multicolumn{4}{|l|}{ Baseline condition } \\
\hline Diabetes mellitus & $11(30.6)$ & $4(20.0)$ & 0.393 \\
\hline Hypertension & $12(33 \cdot 3)$ & $9(45 \cdot 0)$ & 0.388 \\
\hline Heart disease & $8(22.2)$ & $8(40.0)$ & 0.158 \\
\hline Others & $7(19 \cdot 4)$ & $3(15 \cdot 0)$ & 0.677 \\
\hline AKI cause & & & 0.167 \\
\hline Ischemic ATN & $14(38.9)$ & $12(60.0)$ & \\
\hline Infection-related ATN & $11(30.6)$ & $6(30.0)$ & \\
\hline Others & $11(30.6)$ & $2(10.0)$ & \\
\hline CRRT duration, hr & $87.8 \pm 57.0$ & $140.2 \pm 78.9$ & 0.006 \\
\hline Anuria duration, hr & $56.28 \pm 92.6$ & $201.3 \pm 219.8$ & 0.001 \\
\hline Body mass index, $\mathrm{kg} / \mathrm{m}^{2}$ & $23.2 \pm 4.3$ & $20.5 \pm 3.1$ & 0.071 \\
\hline Initial Hb, g/dL & $11.2 \pm 2.3$ & $10.6 \pm 2.8$ & 0.420 \\
\hline Initial CRP, mg/dL & $12.8 \pm 11.9$ & $10.9 \pm 10.9$ & 0.571 \\
\hline Initial Cr, mg/dL & $3.7 \pm 2.1$ & $3 \cdot 9 \pm 2.2$ & 0.801 \\
\hline Initial eGFR, $\mathrm{mL} / \mathrm{min} / 1.73 \mathrm{~m}^{2}$ & $21.1 \pm 11.7$ & $18.9 \pm 11.7$ & 0.502 \\
\hline Final Cr, mg/dL & $0.9 \pm 0.2$ & $1.6 \pm 0.6$ & $<0.001$ \\
\hline Final eGFR, $\mathrm{mL} / \mathrm{min} / 1.73 \mathrm{~m}^{2}$ & $87.3 \pm 23.0$ & $42.9 \pm 12.4$ & $<0.001$ \\
\hline Average MAP, mmHg & $90.0 \pm 9.1$ & $87.5 \pm 10.2$ & 0.346 \\
\hline Volume balance, mL & $536.7 \pm 4,155 \cdot 2$ & $100.6 \pm 4,298.8$ & 0.712 \\
\hline
\end{tabular}

Values are presented as mean $\pm \mathrm{SD}$ or number (\%).

AKI, acute kidney injury; CRRT, continuous renal replacement therapy; ATN, acute tubular necrosis; Hb, hemoglobin; CRP, C-reactive protein; Cr, creatinine; eGFR, estimated glomerular filtration rate; MAP, mean arterial pressure.

et al. [16] analyzed a prospective cohort of AKI patients initiated on CRRT and found that no demographic or laboratory parameter significantly predicted renal recovery in discharged patients. In contrast, a randomized controlled trial comparing intermittent dialysis with CRRT performed by Mehta et al. [17] showed that severity of illness was an important factor in renal recovery. Uehlinger et al. [18] found that pre-existing CKD was an independent predictor of renal recovery. In the recent retrospective study by $\mathrm{Ng}$ et al. [19], the presence of sepsis was included as an independent predictor of renal recovery. These differing results may be attributable to differences in RRT modality, the definition of AKI, or the criteria for renal recovery.

The current study enrolled patients treated with CRRT who manifested severe AKI and cardiovascular instability. There was no difference in underlying disease or laboratory findings between the complete and incomplete renal recovery groups. Multiple regression analysis revealed that age and anuria duration were independent predictors of renal recovery. These findings are consistent with those of Augustine et al. [20], who reported that urine output on day 1 and preserved urine output on day 3 were independently correlated with renal recovery. In that study, the definition of renal recovery was being free from dialysis therapy. The comparison was performed between patients with renal recovery and patients who died or still required maintenance dialysis, which meant that preserved urine volume in the early dialysis period would be associated with cessation of dialysis, regardless of complete recovery to previous renal function. The clinical significance of urine 
Table 2. Multiple logistic regression analysis of factors that increased the risk of incomplete recovery after acute kidney injury

\begin{tabular}{|c|c|c|c|c|c|c|}
\hline \multirow{2}{*}{ Variable } & \multicolumn{2}{|l|}{ Unadjusted } & \multicolumn{2}{|l|}{ Adjusted } & \multicolumn{2}{|l|}{ Fully adjusted } \\
\hline & OR $(95 \% \mathrm{CI})$ & $p$ value & OR $(95 \% \mathrm{CI})$ & $p$ value & OR $(95 \% \mathrm{CI})$ & $p$ value \\
\hline Age, yr & $1.061(1.009-1.116)$ & 0.021 & $1.198(1.063-1.351)$ & 0.003 & $1.231(1.041-1.457)$ & 0.015 \\
\hline Male sex & $0.462(0.152-1.407)$ & 0.174 & & & $0.495(0.062-3.919)$ & 0.505 \\
\hline \multicolumn{7}{|l|}{ Baseline condition } \\
\hline Diabetes & $0.568(0.154-2.096)$ & 0.396 & & & $0.822(0.085-7.920)$ & 0.866 \\
\hline Hypertension & $1.636(0.533-5.019)$ & 0.389 & & & $1.099(0.157-7.716)$ & 0.924 \\
\hline \multicolumn{7}{|l|}{ AKI cause, $\%$} \\
\hline Ischemic ATN & $4.714(0.868-25.612)$ & 0.073 & $10.185(0.905-114.630)$ & 0.060 & $10.073(0.402-252.144)$ & 0.160 \\
\hline Infection-related ATN & $3.000(0.493-18.247)$ & 0.233 & $1.081(0.102-11.469)$ & 0.948 & $0.669(0.029-15.459)$ & 0.802 \\
\hline CRRT duration, hr & $1.012(1.003-1.022)$ & 0.013 & $1.023(1.004-1.043)$ & 0.020 & $1.024(1.000-1.050)$ & 0.052 \\
\hline Anuria duration, $6 \mathrm{hr}$ & $1.038(1.010-1.066)$ & 0.007 & $1.059(1.007-1.114)$ & 0.026 & $1.064(1.001-1.131)$ & 0.047 \\
\hline Initial hemoglobin, g/dL & $0.909(0.724-1.142)$ & 0.414 & & & $0.879(0.592-1.305)$ & 0.523 \\
\hline Initial CRP, mg/dL & $0.986(0.938-1.035)$ & 0.563 & & & $1.005(0.890-1.135)$ & 0.934 \\
\hline Initial eGFR, $\mathrm{mL} / \mathrm{min} / 1.73 \mathrm{~m}^{2}$ & $0.983(0.936-1.033)$ & 0.495 & & & $0.999(0.910-1.097)$ & 0.986 \\
\hline Average MAP, mmHg & $0.972(0.917-1.031)$ & 0.341 & & & $1.012(0.884-1.159)$ & 0.862 \\
\hline Volume balance, $\mathrm{mL}$ & $1.000(1.000-1.000)$ & 0.706 & & & $1.000(1.000-1.000)$ & 0.436 \\
\hline
\end{tabular}

OR, odds ratio; CI, confidence interval; AKI, acute kidney injury; ATN, acute tubular necrosis; CRRT, continuous renal replacement therapy; CRP, C-reactive protein; eGFR, estimated glomerular filtration rate; MAP, mean arterial pressure.

output in renal recovery was somewhat different from our study, in which urine output might be a prognostic marker even in AKI patients who did not need further dialysis therapy.

Several studies have evaluated the clinical implications of urine output monitoring in patients with CRRT. Suzuki et al. [21] investigated whether urine output was associated with the survival of AKI patients receiving CRRT in a small, randomized trial that used urine output as a criterion for starting CRRT after coronary bypass graft surgery. They compared patient survival between the early start group, which initiated dialysis therapy when urine volume decreased to less than 30 $\mathrm{mL} / \mathrm{hr}$ for 3 consecutive hours, and the late start group, which delayed dialysis therapy until urine volume reached less than $20 \mathrm{~mL} / \mathrm{hr}$ for 2 consecutive hours. The 14-day survival was significantly superior in the early start group, suggesting that urine volume was a good indicator for starting CRRT. Another retrospective study, by $\mathrm{Oh}$ et al. [22], explored the impact of 6-hour urine volume immediately before CRRT start on the survival of AKI patients. They found that 28-day survival rates were significantly higher in the nonoliguric group (6- hour urine output $>107 \mathrm{~mL}$ ) than in the oliguric group (6-hour urine output $\leq 107 \mathrm{~mL}$ ). As well as a criterion for CRRT start, urine volume would also be applicable as a predictor of redialysis after CRRT weaning. Wu et al. [23] compared the characteristics of patients with successful and failed weaning of postoperative RRT. A longer duration of dialysis, multiple organ failure, urine output $<100 \mathrm{~mL}$ for 8 hours on the first dialysis day, and age over 65 years were independent predictors for resuming dialysis within 30 days. In other studies by Uchino et al. [24] and Heise et al. [25], increased urine output was also independently associated with CRRT discontinuation and could thus predict the restoration of kidney function. Taken together with our study, these results indicate that urine output monitoring is a simple but useful clinical method of discriminating the proper timing of CRRT initiation, weaning, and further predicting the recovery of renal function after CRRT.

Measurement of urine output is practical in AKI patients; several AKI guidelines have introduced it as a diagnostic criterion along with $\mathrm{Cr}[1,2]$. A decrease in urine output for a certain duration was considered to be a sensitive and easily discernible means of identifying 
AKI patients. However, urine volume may be influenced by volume status, diuretic dose, and hemodynamic stability $[26,27]$. Furthermore, the nonoliguric type of AKI can be associated with a relatively normal urine output [28]. Despite these limitations, previous studies have shown that urine volume has an independent association with outcomes in AKI patients, regardless of other clinical parameters $[20,24]$. It is a remarkable finding of our study that, although urine volume itself is an important marker of AKI, the duration of decreased urine volume could also reflect renal injury and thus predict renal recovery. To validate our result, further prospective studies evaluating the impact of anuria duration on long-term renal outcomes in AKI patients receiving CRRT would be needed.

The findings of the current study are interesting; we were unable to find similar results in other studies. However, several limitations should be addressed. First, the number of patients was relatively small. This was due to the high mortality and morbidity in severe AKI patients. Nevertheless, the multivariate analysis identified duration of anuria as a statistically significant independent factor. Second, the follow-up duration may have been too short to determine recovery of renal function. Severe AKI cases take longer to return to baseline renal function [29]; however, the severity of AKI itself is known to be associated with CKD progression [30]. Third, the impact of anuria on patient survival could not be evaluated because only surviving patients after CRRT were included in the analysis. Despite these limitations, a compelling outcome of our study is the identification of anuria duration, a simple practical parameter, as a good prognostic marker in a relatively heterogeneous AKI patient group.

In conclusion, the renal outcome of severe AKI requiring CRRT was poor even in patients with previously normal renal function. Long-lasting insufficient urine volume predicted decreased renal function after recovery from severe AKI requiring CRRT. Long-term monitoring of renal function is warranted, especially in severe AKI patients who are old and have a long duration of anuria.

\section{KEY MESSAGE}

1. The renal outcome of severe acute kidney injury (AKI) patients requiring continuous renal replacement therapy (CRRT) was poor, even in patients with previously normal renal function.

2. Long-lasting insufficient urine volume predicted decreased renal function after recovery from severe AKI requiring CRRT.

\section{Conflict of interest}

No potential conflict of interest relevant to this article was reported.

\section{Acknowledgments}

This work was supported by a grant from the Biomedical Research Institute, Kyungpook National University Hospital (2015).

\section{REFERENCES}

1. Bellomo R, Ronco C, Kellum JA, Mehta RL, Palevsky P; Acute Dialysis Quality Initiative workgroup. Acute renal failure: definition, outcome measures, animal models, fluid therapy and information technology needs: the Second International Consensus Conference of the Acute Dialysis Quality Initiative (ADQI) Group. Crit Care 2004;8:R204-R212.

2. Mehta RL, Kellum JA, Shah SV, et al. Acute Kidney Injury Network: report of an initiative to improve outcomes in acute kidney injury. Crit Care 2007;11:R31.

3. Uchino S, Kellum JA, Bellomo R, et al. Acute renal failure in critically ill patients: a multinational, multicenter study. JAMA 2005;294:813-818.

4. Ishani A, Xue JL, Himmelfarb J, et al. Acute kidney injury increases risk of ESRD among elderly. J Am Soc Nephrol 2009;20:223-228.

5. Wald R, Quinn RR, Luo J, et al. Chronic dialysis and death among survivors of acute kidney injury requiring dialysis. JAMA 2009;302:1179-1185.

6. Pannu N, James M, Hemmelgarn B, Klarenbach S; Alberta Kidney Disease Network. Association between AKI, recovery of renal function, and long-term outcomes after hospital discharge. Clin J Am Soc Nephrol 2013;8:194-202. 
7. Hsu CY, Ordonez JD, Chertow GM, Fan D, McCulloch CE, Go AS. The risk of acute renal failure in patients with chronic kidney disease. Kidney Int 2008;74:101-107.

8. Swets JA. Measuring the accuracy of diagnostic systems. Science 1988;240:1285-1293.

9. Hsu CY, McCulloch CE, Fan D, Ordonez JD, Chertow GM, Go AS. Community-based incidence of acute renal failure. Kidney Int 2007;72:208-212.

10. Hsu RK, McCulloch CE, Dudley RA, Lo LJ, Hsu CY. Temporal changes in incidence of dialysis-requiring AKI. J Am Soc Nephrol 2013;24:37-42.

11. Coca SG, Singanamala S, Parikh CR. Chronic kidney disease after acute kidney injury: a systematic review and meta-analysis. Kidney Int 2012;81:442-448.

12. Jones J, Holmen J, De Graauw J, Jovanovich A, Thornton $\mathrm{S}$, Chonchol M. Association of complete recovery from acute kidney injury with incident CKD stage 3 and allcause mortality. Am J Kidney Dis 2012;60:402-408.

13. Rifkin DE, Coca SG, Kalantar-Zadeh K. Does AKI truly lead to CKD? J Am Soc Nephrol 2012;23:979-984.

14. Schmitt R, Coca S, Kanbay M, Tinetti ME, Cantley LG, Parikh CR. Recovery of kidney function after acute kidney injury in the elderly: a systematic review and meta-analysis. Am J Kidney Dis 2008;52:262-271.

15. Schiffl H. Renal recovery from acute tubular necrosis requiring renal replacement therapy: a prospective study in critically ill patients. Nephrol Dial Transplant 2006;21:1248-1252.

16. Allegretti AS, Steele DJ, David-Kasdan JA, Bajwa E, Niles JL, Bhan I. Continuous renal replacement therapy outcomes in acute kidney injury and end-stage renal disease: a cohort study. Crit Care 2013;17:R109.

17. Mehta RL, McDonald B, Gabbai FB, et al. A randomized clinical trial of continuous versus intermittent dialysis for acute renal failure. Kidney Int 2001;60:1154-1163.

18. Uehlinger DE, Jakob SM, Ferrari P, et al. Comparison of continuous and intermittent renal replacement therapy for acute renal failure. Nephrol Dial Transplant 2005;20:1630-1637.

19. Ng KP, Chanouzas D, Fallouh B, Baharani J. Short and long-term outcome of patients with severe acute kid- ney injury requiring renal replacement therapy. QJM 2012;105:33-39.

20. Augustine JJ, Sandy D, Seifert TH, Paganini EP. A randomized controlled trial comparing intermittent with continuous dialysis in patients with ARF. Am J Kidney Dis 2004;44:1000-1007.

21. Suzuki H, Kanno Y, Kaneko K, et al. Comparison of the effects of angiotensin receptor antagonist, angiotensin converting enzyme inhibitor, and their combination on regression of left ventricular hypertrophy of diabetes type 2 patients on recent onset hemodialysis therapy. Ther Apher Dial 2004;8:320-327.

22. Oh HJ, Shin DH, Lee MJ, et al. Urine output is associated with prognosis in patients with acute kidney injury requiring continuous renal replacement therapy. J Crit Care 2013;28:379-388.

23. Wu VC, Ko WJ, Chang HW, et al. Risk factors of early redialysis after weaning from postoperative acute renal replacement therapy. Intensive Care Med 2008;34:101-108.

24. Uchino S, Bellomo R, Morimatsu H, et al. Discontinuation of continuous renal replacement therapy: a post hoc analysis of a prospective multicenter observational study. Crit Care Med 2009;37:2576-2582.

25. Heise D, Gries D, Moerer O, Bleckmann A, Quintel M. Predicting restoration of kidney function during CRRT-free intervals. J Cardiothorac Surg 2012;7:6.

26. Pinsky MR, Brophy P, Padilla J, Paganini E, Pannu N. Fluid and volume monitoring. Int J Artif Organs 2008;31:111126.

27. Bagshaw SM, Bellomo R, Kellum JA. Oliguria, volume overload, and loop diuretics. Crit Care Med 2008;36(4 Suppl):S172-S178.

28. Avila MO, Zanetta DM, Abdulkader RC, Yu L, Burdmann EA. Urine volume in acute kidney injury: how much is enough? Ren Fail 2009;31:884-890.

29. Myers BD, Moran SM. Hemodynamically mediated acute renal failure. N Engl J Med 1986;314:97-105.

30. Chawla LS, Amdur RL, Amodeo S, Kimmel PL, Palant CE. The severity of acute kidney injury predicts progression to chronic kidney disease. Kidney Int 2011;79:1361-1369. 